\title{
Multispectral IR thermotransmittance technique for temperature measurement
}

\author{
by C. Pradere*, M. Ryu**, A. Sommier*, M. Romano***, J. Morikawa** and J.C. Batsale* \\ * I2M TREFLE, UMR CNRS 5295, Esplanade des Arts et Métiers, 33405 Talence, France, c.pradere@i2m.u- \\ bordeaux1.fr, romano@enscpb.fr, a.sommier@2m.u-bordeaux1.fr, jc.batsale@2m.u-bordeaux1.fr. \\ ** Tokyo Institute of Technology, Department of Organic and Polymeric Materials, Tokyo, Japan, \\ ryu.m.ab@m.titech.ac.jp, morikawa.j.aa@m.titech.ac.jp. \\ romano@enscbp.fr \\ *** Epsilon Fahrenheit, ENSAM, Esplanade des Arts et Métiers, F-33405 Talence Cedex, France,
}

\section{Abstract}

The goal of this work is to reinforce the recent observed giant sensitivity of thermotransmittance technique in far infrared wavelength (millimetric one) by transposing such technique in the mid infrared region. Here, the thermotransmittance coefficient determined at $\mathbb{R}$ multiw avelength from 3 to $5 \mu \mathrm{m}$ and for several semi-transparent media (Silicon, Sapphire) is reported. The major result is the sensitivity dependency of the transmittance coefficient to the $\mathbb{R}$ spectrum of studied material as well as the good relative sensitivity. As a consequence tomographic and non-contact multispectral temperature measurement could be considered $w$ ith this method.

\section{Introduction}

For years, thermoreflectance techniques have been used to perform thermal imaging of heterogeneous materials [1]. Working with visible wavelengths enables to achieve nanometric spatial resolutions. Furthermore, the use of ultra-fast lasers allow s picosecond time resolution. Tessier et al. [2] have show $n$ that the nature of the material under investigation associated to the wavelength play a crucial role on the temperature measurement sensitivity and the global range for the coefficient is within $10^{-4}$ to $10^{-6} \mathrm{~K}^{-1}$. In the last years, THz 2D imaging (and even 3D tomography) techniques for semiconductors and insulating materials have been discussed and demonstrated [3], they turn to be a well-adapted tool for their characterization. THz waves are defined as a very far $\mathbb{R}$ wavelength covering a spectral range from $30 \mu \mathrm{m}$ to $3 \mathrm{~mm}$. Based on works previously reported [4], the thermoreflectance technique from visible to $\mathrm{THz}$ wavelengths has been extended. Thus, the ability of those millimetric radiations to pass through a thick plate of Teflon opaque to visible wavelengths and perform transient temperature measurements of metallic and semiconductor materials has been demonstrated. By monitoring the temperature of the sample, the estimation of the thermoreflectance coefficient of aluminum, copper and silicon has been done. In this work, the giant sensibility is highlighted through the thermoreflectance coefficients whose values are 100 times higher in the $\mathrm{THz}$ domain compared to the optimized coefficients previously reported for visible wavelength. In this paper, the thermotransmittance is reported as the mean intensity variation linked to temperature variation of the incident radiation light passing through a semi-transparent media in the $\mathbb{R}$ band.

\section{Experimental set-up}

The experimental set-up used in this study and schematically presented figure 1 is composed of a high-speed infrared (IR) camera (Phoenix Indigo) and a grating monochromator to select the excitation wavelength (CT-10, JASCO Corporation, Tokyo, Japan). The beam emitted from the slit (output of the monochromator, Figure 1-1) is monochromatic. The source light is transmitted from the monochromator to a parabolic mirror (Figure 1-2) to guide it through the sample (Figure 1-4); the transmitted light is recorded by the IR camera used as a sensor (Figure 1-6) to perform the micro-scale thermography. The camera has an indium-antimony ( $\mathrm{InSb}$ ) detector composed of a focal plane array (FPA matrix $320 \mathrm{x}$ 256) that act as independent sensors, and the simultaneous measurement of 81920 spots can be performed using the snapshot mode in $1 \mathrm{~ms}$. The camera is equipped $w$ ith an original designed microscopic lens (from Tokyo Tech Institute, Japan) with a magnification of $7.5 \mathrm{X}$ and a spatial resolution of $4.1 \mu \mathrm{m}$ per pixel at 5 microns. The frame rate acquisition was set to $200 \mathrm{~Hz}$. The infrared spectra were acquired using the synchronization of the monochromator and the $\mathbb{R}$ camera by using a mechanical chopper with homemade LabVIEW softw are to control the acquisition frequency and to scan the wavelength over the domain of interest. As a consequence, w ith the set-up described in figure 1, and as already demonstrated in previous papers [5-6] the acquired signal is based on two images techniques which are function of several contributions as follow:

$$
I(x, y, \lambda, T, \Gamma)=S(x, y, \lambda, T, \Gamma)+\mathrm{E}(x, y, \lambda, T)
$$


Where $I$, is the global acquired intensity corresponding to the sum of the spectrum one, $S$ and the proper emission one, $E$ (w hen the mechanical chopper is open) and only the proper emission $E$ (when the chopper is off). By a simple difference betw een the two signals both thermal and spectral images could be retrieved.

In order to impose the temperature to prove the thermal dependency of the spectrum transmission, the sample is $w$ arming up in a heating stage. The sample holder show $n$ in figure 1 is a S84 microscope heat stage (ST Japan) with two $\mathrm{BaF}_{2}$ windows at the top and bottom of the device to enable the observation in transmission mode, and inside the enclosure, the temperature is controlled $w$ ith $0.1^{\circ} \mathrm{C}$ precision. For the experimentation presented here, the heating stage temperature is fixed at several temperatures $\left(21,80\right.$ and $\left.140^{\circ} \mathrm{C}\right)$ and a complete spectrum of the several substrates $w$ as acquired.

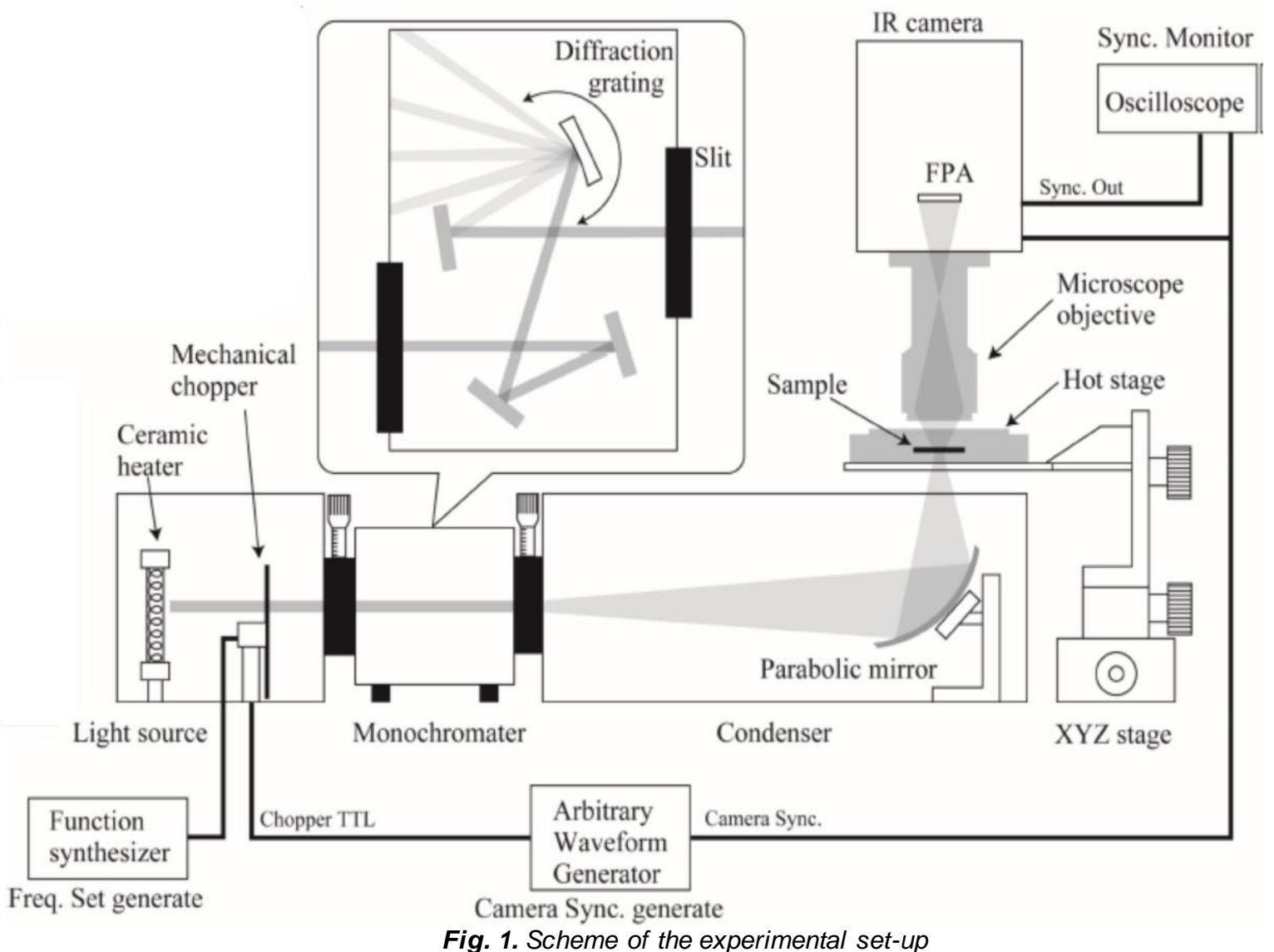

Fig. 1. Scheme of the experimental set-up

\section{Thermotransmittance expression and measurement procedure}

By transposition of the definition of the thermoreflectance formulation [2], the thermotransmittance of an optical field could be formulated as follow:

$$
\frac{S(x, y, \lambda, T)-S\left(x, y, \lambda, T_{0}\right)}{S\left(x, y, \lambda, T_{0}\right)}=\frac{\partial S(x, y, \lambda, T)}{S\left(x, y, \lambda, T_{0}\right) \partial T}\left(T(x, y)-T_{0}(x, y)\right)=\tau(x, y, \lambda)\left(T(x, y)-T_{0}(x, y)\right)
$$

Where $S$ corresponds to the spectrum signal measured in transmission mode and $T$ is the imposed temperature of the heating stage measured by using a thermocouple.

As a consequence, during a steady state regime corresponding to imposed temperatures of 21,80 and $140^{\circ} \mathrm{C}$ both $\mathbb{I R}$ spectrum and temperature measured by proper emission are acquired, see Eq. (1). This huge amount of data, represented figure 2 corresponds to hypercube of thermospectral data. In this paper, the transmission dependency of the IR spectrum to temperature variation, also called thermotransmission will be highlighted. To explain that, a set of results based on the data obtained on one pixel of the images will be used to demonstrate this phenomenon. 


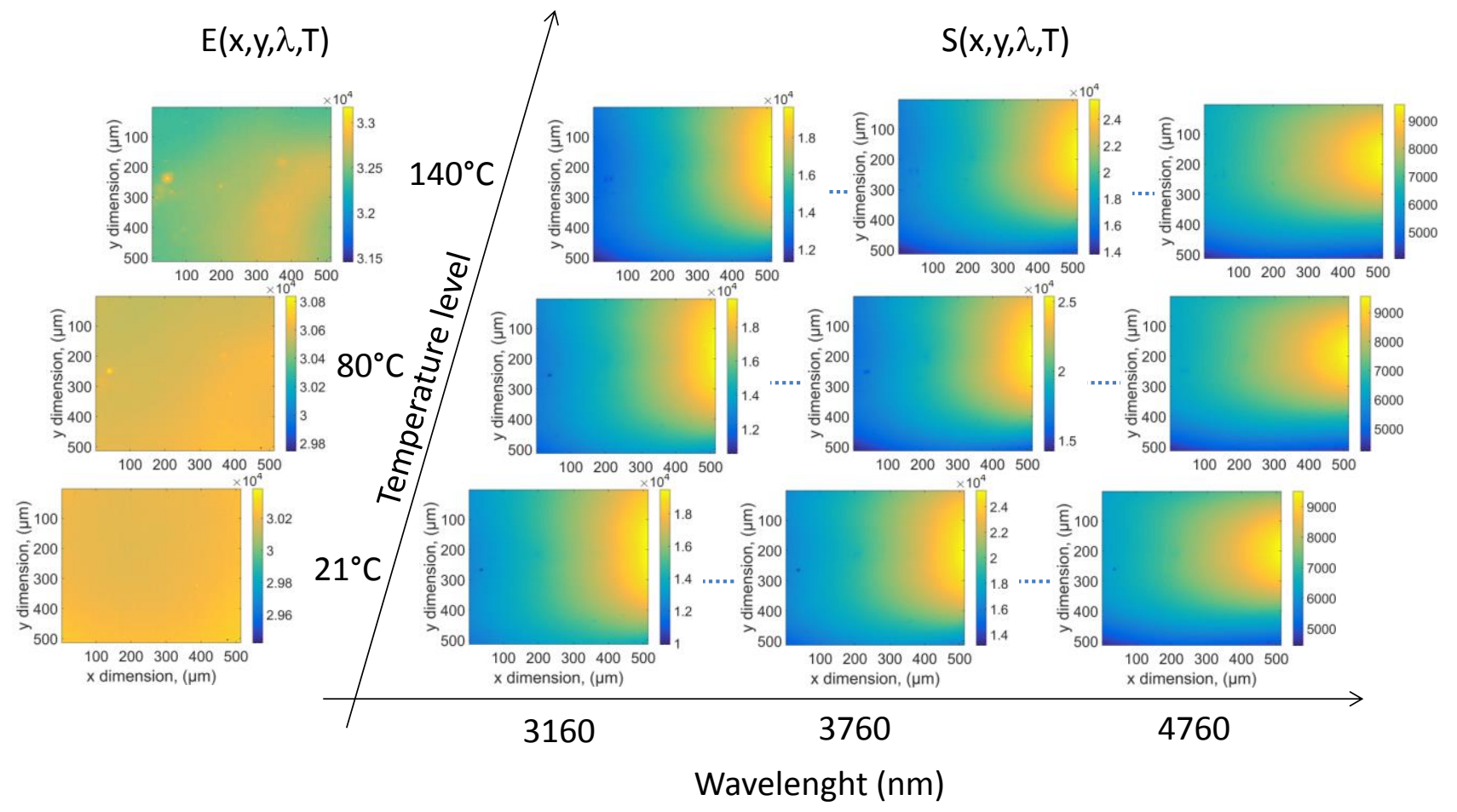

Fig. 2. Example of recorded data in Digital Level $(D L)$ as function of temperatures and wavelengths: cube of measured temperatures in proper emission $E(x, y, \lambda, T)$ and hypercube of IR spectrum in transmission mode $S(x, y, \lambda, T)$.

Based on the hypercube figure 2, the spectrum and temperature measured on one pixel (located in the center of the images) as function of the wavelength and for the several imposed temperatures are represented:
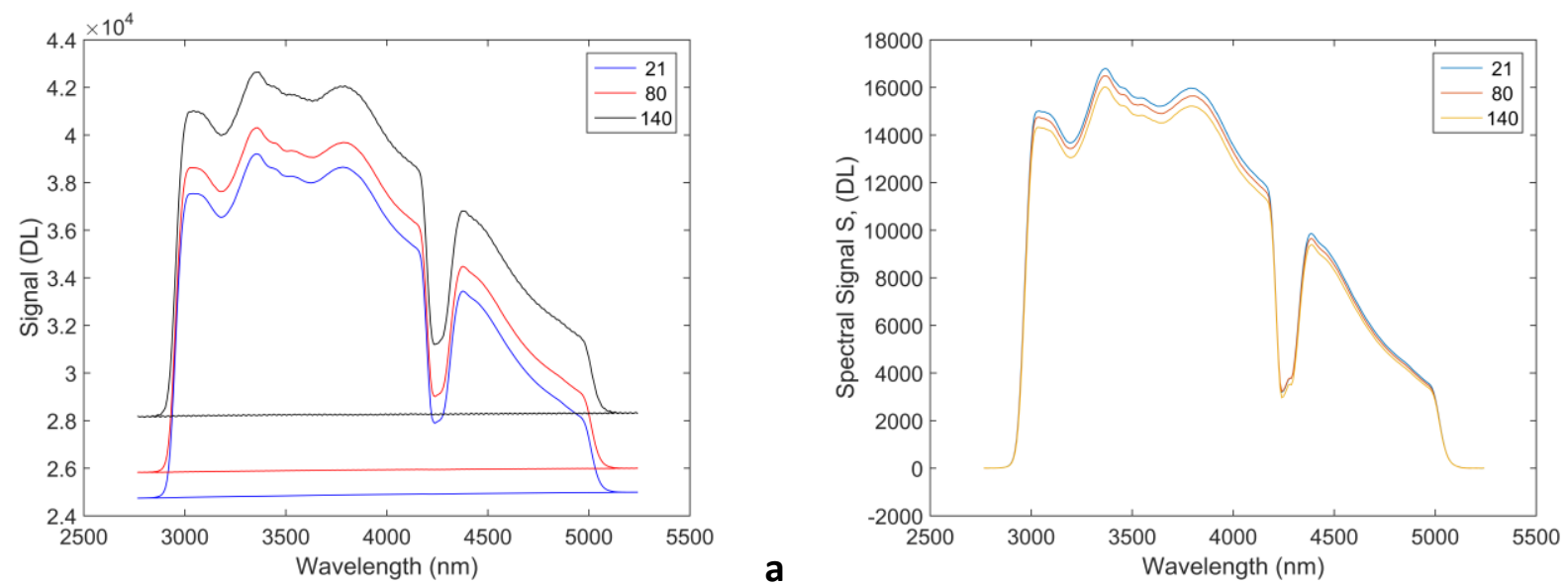

Fig. 3. Measurement on Saphhire substrate for one pixel (xi,yi) of the images represented figure 1 as function of the wavelength for several temperatures: a), measured intensity $I(x i, y i, \lambda, T)$ in transmission mode and temperature by proper emission $E(x i, y i, \lambda, T)$ and $b)$, Spectrum $S(x i, y i, \lambda, T)$ extracting from Eq.2.

Once can be observed that even in a steady state mode, and due to the thermal regulation of the heating stage, the baseline (corresponding to the proper emission) is not perfectly constant and especially for the higher temperature of $140^{\circ} \mathrm{C}$ (black curve figure 3.a), the effect of the regulation affects the data. Another important point is that the baseline is a function of the temperature. As a consequence, the spectrum in figure 3.a seems to have a very important thermal dependency whereas they are mainly affected by the proper emission dependency. This illustrates the first interest of the two images technique [5-6] that is to have the possibility to subtract this thermal dependent baseline. Then, by making this subtraction, only the spectrum as function of temperature can be plotted in figure 3.b. Finally, it can be clearly observed in figure 3.a that for three different imposed temperatures to the substrate (here the Sapphire was taken as example), it seems that the spectrum varied of few percent in amplitude and not completely constant depending the w avelength. 


\section{Results and discussion}

To demonstrate this thermotransmission dependency, and according to Eq. (2), an inverse method (GaussMarkov algorithm) based on linear regression is applied to fit the experimental data and retrieve the thermotransmittance coefficient $\tau\left(K^{1}\right)$. The results see figures 4,5 and 6 for several semi-transparent to $\mathbb{R}$ media are represented. Once can be noticed that all this experimentation $w$ as not done under vacuum and that around $4250 \mathrm{~nm}$ where the $\mathbb{R}$ signal is strongly sensitive to the $\mathrm{CO}_{2}$ presents inside air, the measured data are perturbed. Another type of study will be performed for several gases and also under vacuum in a further paper.

\subsection{Measurements on Sapphire}

First, figure 4.a, the transmission of the Sapphire is represented as function of the wavelength and for several imposed temperatures. It could be interesting to notice that this sample is a good semi-transparent to IR domain, with an average value of 0.75 and that the semi-transparency tends to decrease when the temperature of the substrate increases. From this, and based on the Eq. (2), the spectrum ratio $\Delta S / S_{0}$ was calculated and the linear regression between this ratio and the temperature imposed and measured by the thermocouple was performed in figure 4.b. The Coefficient of ThermoTransmission (CTT) estimated as a function of the wavelength is represented as w ell as a zoom of the linear fit for a given wavelength. As explained before, excepted around the $\mathrm{CO}_{2}$ dependency of $\mathbb{R}$, the spectral thermo-dependency inside the substrate of Sapphire could be clearly seen with a coefficient around $6.10^{-4} \mathrm{~K}^{-1}$. Moreover, this estimation of the CTT coefficient could be realized overall the pixels of the hypercubes (figure 2) and the obtained coefficients, figure 4.d, could be compared to the temperature measured in DL by the $\mathbb{R}$ camera in proper emission, see figure 4.c. In this case, it seems that both measured techniques are in quite good agreements and show that the temperature fields are homogeneous $w$ ith the same temperature jumps due to optical artefacts (emissivity or dust or sample defect).

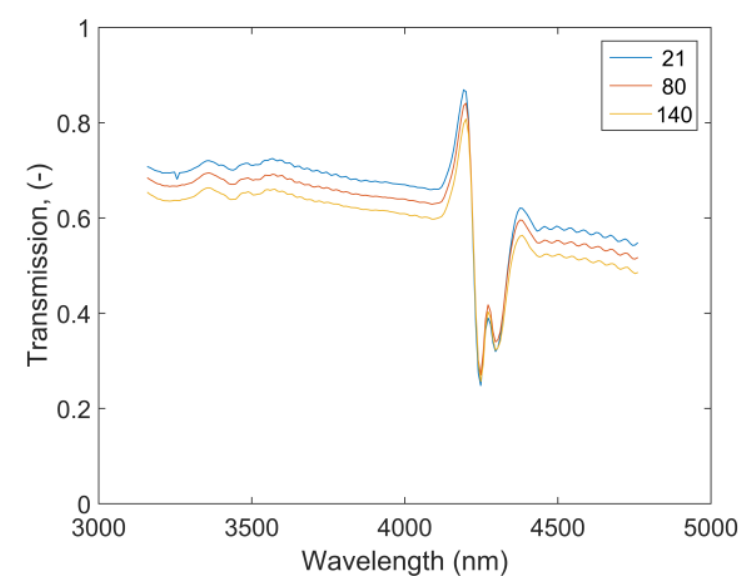

a
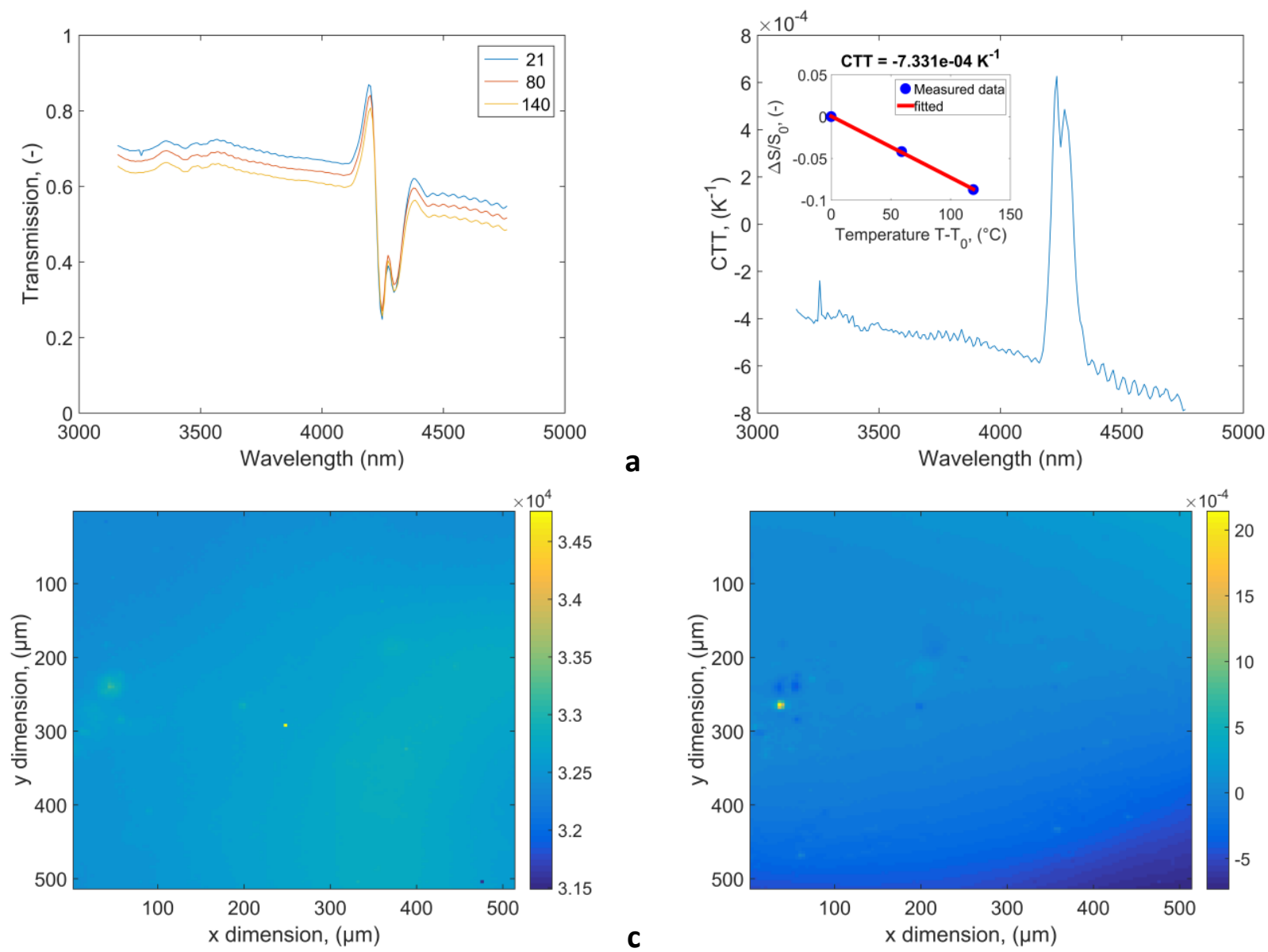

Fig. 4. Results on Sapphire substrate: a), Transmission of one pixel of the image as function of wavelength at several temperatures, b), estimated Coefficient of ThermoTransmission (CTT) as function of the wavelength and zoom of the fitted data at $4500 \mathrm{~nm}, \mathrm{c}$ ), measured temperature in $D L$ by proper emission at $140^{\circ} \mathrm{C}$ and d), Estimated CTT in $\mathrm{K}^{1}$ 


\subsection{Measurements on Silicon}

Finally figure 5.a, the transmission of the Silicon is represented as function of the wavelength and for several imposed temperatures. It could be interesting to notice that this particular doped silicon wafer is not a good semitransparent to $\mathbb{I R}$ wavelength, with an average value of 0.015 and that the semi-transparency tends to decrease $w$ hen the temperature of the substrate increases and with lot of noises and difficult for the maximum imposed temperature of $140^{\circ} \mathrm{C}$. From this, and based on the Eq. (2), the spectrum ratio $\Delta S / S_{0}$ w as calculated and the linear regression betw een this ratio and the temperature imposed and measured by the thermocouple was performed in figure 5.b. The Coefficient of ThermoTransmission (CTT) estimated as function of the wavelength is represented as well as a zoom of the linear fit for a given wavelength. As already explained, excepted around the $\mathrm{CO}_{2}$ dependency of $I R$, the spectral thermodependency inside the substrate of Silicon could be clearly seen with a coefficient around $3.10^{-3} \mathrm{~K}^{-1} \mathrm{w}$ hich is one order of magnitude more important than the two previous samples. This explains why despite the poor transmission we succeed in thermotransmission measurements. Moreover, this estimation of the CTT coefficient could be realized overall the pixels of the hypercubes (figure 2) and the obtained coefficients, figure 5.d, could be compared to the temperature measured in DL by the IR camera in proper emission, see figure 5.c. As for the Sapphire, it seems that for such Silicon substrate, both measured techniques are in quite good agreements and show that the temperature fields are not homogeneous to the doped particle inside the Silicon, with the same temperature jumps due to optical artefacts (emissivity or sample defect). Moreover, it can be observed that in such an inhomogeneous sample, the CTT could take positive or negative values as a function of the spectral response of each constituent.
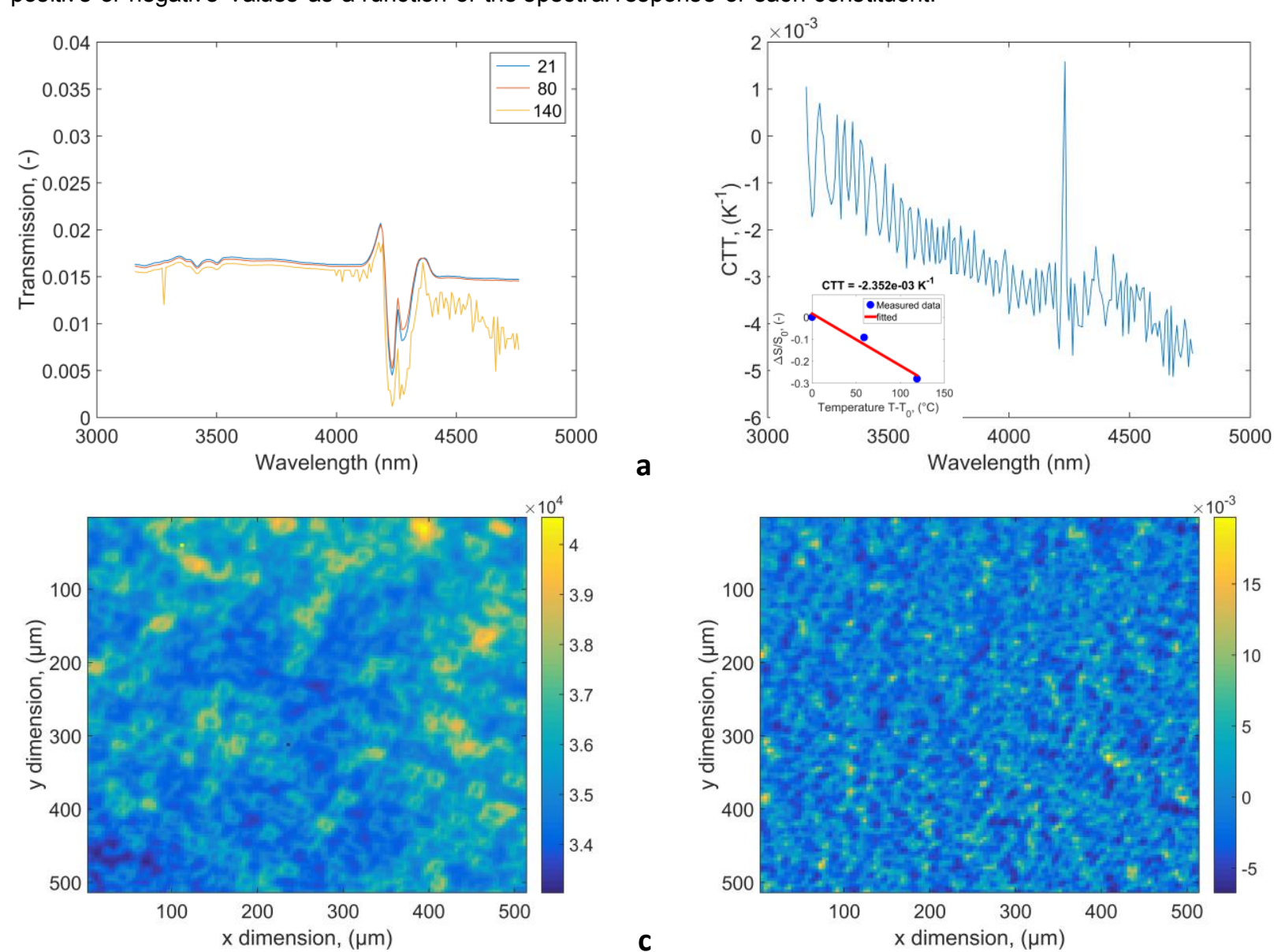

a

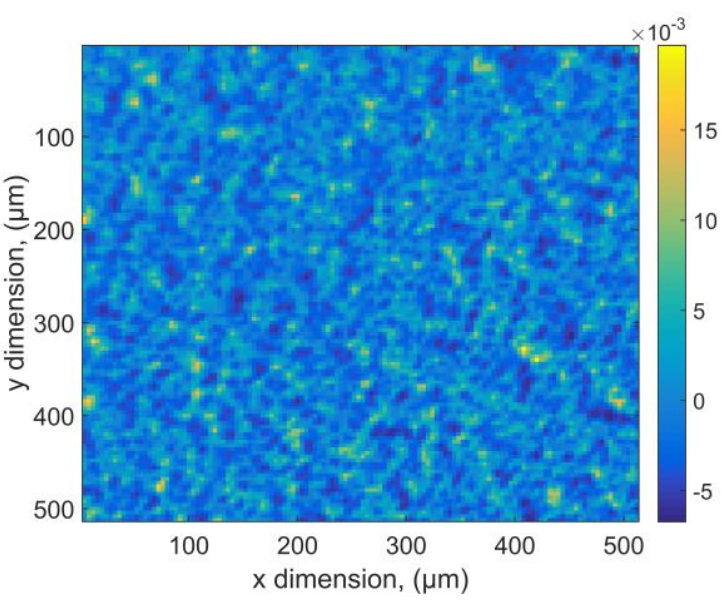

Fig. 5. Results on Silicon substrate: a), Transmission of one pixel of the image as function of wavelength at several temperatures, b), estimated Coefficient of ThermoTransmission (CTT) as function of the wavelength and zoom of the fitted data at $3500 \mathrm{~nm}, \mathrm{C}$ ), measured temperature in $\mathrm{DL}$ by proper emission at $140^{\circ} \mathrm{C}$ and d), Estimated CTT in $\mathrm{K}^{1}{ }^{1}$

\section{Conclusion}

To conclude this preliminary work, it seems that multispectral thermotransmittance technique could be a powerful tool to measure in-situ and without any contact the temperature of solid semi-transparent media in $\mathbb{I R}$ wavelength by using IR spectrum. In this paper, the sensitivity dependence according to the wavelength and the 
chemical compound of three samples has been demonstrated. Moreover, even if this thermal dependency is proved, complementary study should be performed especially the air influence, the optical shape of the incident beam (confocal, or parallel...). Moreover, the thermal dependency of the $\mathbb{R}$ spectrum as a function of the chemical composition of the substrate should be clarified, especially if the thermal sensitivity is a function of the derivative of the raw spectrum with maximum sensitivity at the inflexion point or more proportional to the raw (at room temperature) spectrum.

\section{ACKNOWLEDGEMENT}

This work was supported by "Défi Imagin" project of French CNRS and also partly supported by the Precise Measurement Technology Promotion Foundation (PMTP-F) in Japan. J.M. acknow ledges the support of JSPS KAKENHI Grant No. 16K06768 and 25420752. C.P. and M.R. are grateful for partial support by Tokyo Institute of Technology via Top Global University project of JSPS. C.P. thanks to the travel support by General Incorporated Foundation for Promotion of Textile Technology (FTPP).

\section{REFERENCES}

[1] E. Matatagui, A. G. Thompson, and M. Cardona, "Thermoreflectance in semiconductors," Phys. Rev., Vol 176, No3, 950-960 (1968).

[2] G. Tessier, G. Jerosolimski, S. Hole, D. Fournier, and C. Filloy, "Measuring and predicting the thermoreflectance sensitivity as a function of wavelength on encapsulated materials", Rev. Sci. Inst., Vol $74, \mathrm{~N}^{\circ}$ 1, 2003.

[3] B. B. Hu, M. C. Nuss, and B. B. Hu, "Imaging with terahertz waves," Opt. Lett. 20(16), 1716-1718 (1995).

[4] C. Pradere, J.-P. Caumes, S. Benkhemis, G. Pernot, E. Palomo, S. Dilhaire, and J.C. Batsale, (2014), "Thermoreflectance Temperature measurement with millimeter wave", Review of Scientific Instrument, ISSN: 0034-6748, 85(06), 4904-4906.

[5] M. Romano, C. Ndiaye, A. Duphil, A. Sommier, J. Morikaw a, J. Mascetti, J.C. Batsale, L. Servant, C. Pradere, Infrared Physics \& Technology, 2015, 68, 152-158.

[6] M. Romano, M. Ryu, J. Morikaw a, J.C. Batsale, C. Pradere , Infrared Physics \& Technology, 2016, 76, 65-71. 